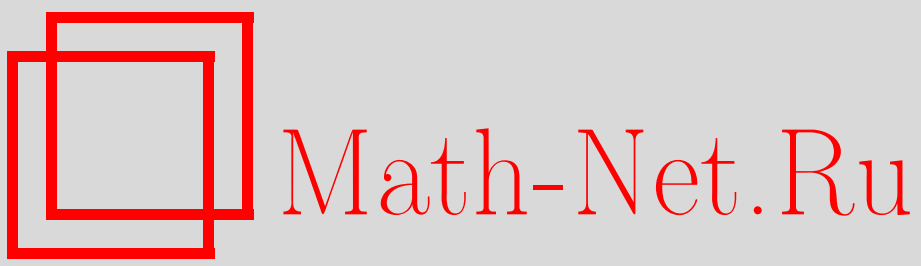

В. И. Юдович, Теорема о неявной функции для косимметрических уравнений, Матем. заметки, 1996, том 60, выпуск 2, 313-317

DOI: https://doi.org/10.4213/mzm1832

Использование Общероссийского математического портала MathNet.Ru подразумевает, что вы прочитали и согласны с пользовательским соглашением

http://www. mathnet.ru/rus/agreement

Параметры загрузки:

IP : 54.157 .27 .8

26 апреля 2023 г., 08:00:39

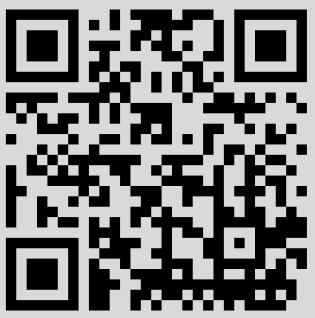




\section{ТЕОРЕМА О НЕЯВНОЙ ФУНКЦИИ ДЛЯ КОСИММЕТРИЧЕСКИХ УРАВНЕНИЙ}

\section{В.И. Юдович}

Косимметрия векторного поля на многообразии, введенная в [1], может служить естественной причиной существования непрерывного семейства равновесий. Такое семейство не является, вообще говоря, орбитой группы симметрии динамической системы, поскольку его точки, в отличие от равновесий орбиты, сохраняют индивидуальность - у каждой свой спектр устойчивости [2].

В этой работе дано общее определение косимметрии. В частности, косимметрией сечения векторного расслоения над многообразием называется сечение сопряженного расслоения, аннулирующее данное сечение в каждой точке многообразия.

Косимметрическая версия теоремы о неявной функции (теорема 1 , простейший вариант см. в [1]) дает достаточные условия существования у операторного уравнения со многими косимметриями локального подмногообразия решений в окрестности известного решения.

При определенных условиях невырожденности возможно гладкое продолжение по параметру как локальных подмногообразий решений (теорема 2), так и глобальных (предложение 2). При этом бифуркация глобальных подмногообразий решений носит локальный характер - начинается в некоторой точке подмногообразия.

1. Косимметрия. Пусть $X, Y$-вещественные банаховы пространства, и опеpaтор $F: X \rightarrow Y$ действует из $X$ в $Y$. Назовем оператор $L: X \rightarrow Y^{*}$ косимметрией оператора $F$, если для всех $x \in X$

$$
(F x, L x)=0 \text {. }
$$

Здесь $($,$) - внутреннее произведение вектора из Y$ и линейного функционала из $Y^{*}$. Мультикосимметрией назовем семейство косимметрий

$$
L=\left\{L_{m}\right\}_{m \in M}
$$

с произвольным множеством индексов $M$.

Рассмотрим уравнение

$$
F x=0
$$

с оператором $F$, допускающим косимметрию $L$. Его решение $x_{0}$ назовем косимметричным, если $L x_{0}=0$.

ПРЕДЛОЖЕНИЕ 1. Пусть оператор $F: X \rightarrow Y$ u его косимметрия $L: X \rightarrow Y^{*}$ дифферениируемь в точке $x \in X$. Тогда выполняется равенство

$$
F^{\prime *}(x) L x+L^{\prime *}(x) F x=0 .
$$

Здесь элемент $F x \in Y$ отохдествляется $c$ его образом во втором сопряженном пространстве $Y^{* *}$ при каноническом вложении.

Работа выполнена при поддержке Российского фонда фундаментальных исследований, грант № 93-013-17337, Международного научного фонда, гранты NRQ000 и NRQ300, и National Scientific Foundation (USA), грант DMS-9300752. 
СлЕДСТВИЕ 1. Некосимметричное решение $x_{0}$ вырожденно: оператор $F^{\prime}\left(x_{0}\right)$ необратим. Ядро $\operatorname{ker}{F^{\prime *}}^{\prime *}\left(x_{0}\right)$ его сопряженного нетривиально: $F^{\prime *}\left(x_{0}\right) L x_{0}=0$.

Это следствие остается верным и в том случае, когда оператор $L$ лишш непрерывен в точке $x_{0}$.

Равенство (4) получаем, дифференцируя (1). Его интересно сравнить с уравнением

$$
F^{\prime *}(x) L x+L^{\prime}(x) F x=0,
$$

которому удовлетворяет пфафффова форма $L: X \rightarrow X^{*}$, инвариантная относительно потока $\exp t F$, порождаемого автономньм дифференциальным уравнением $\dot{x}=F x$ на $X$. Уравнение (5) тоже влечет вырожденность решения $x_{0}$ уравнения (3), если $L x_{0} \neq 0$, и при общих условиях приводит к существованию непрерьвной ветви решений.

Мульикосимметрия (2) индуцирует кораспределение на паре пространств $X, Y:$ каждой точке $x \in X$ сопоставляется подпространство $Y_{* x}=Y_{* x}(L)=$ Clos $\operatorname{Lin}\left\{L_{m} x\right\}_{m \in M}$, а также и распределение - точке $x \in X$ ставится в соответствие аннулятор $\bar{Y}_{x}=Y_{* x}^{\perp} \subset Y$ подпространства $Y_{* x}$ :

$$
\bar{Y}_{x}=\left\{y \in Y: \forall y_{*} \in Y_{* x} \mid\left(y, y_{*}\right)=0\right\}
$$

Точку $x_{0} \in X$ назовем регулярной точкой мультикосимметрии (2), если в некоторой ее окрестности можно определить семейство проекторов $\bar{P}_{x}: Y \rightarrow Y$, $\operatorname{im} \bar{P}_{x}=\bar{Y}_{x}$, непрерьвно зависящее от $x$ по операторной норме. Вводя дополнительный проектор $P_{x}=I-\bar{P}_{x}$ и сопряженные проекторы $\bar{P}_{x}^{*}$ и $P_{x}^{*}$, приходим к прямьм разложениям

$$
Y=Y_{x} \oplus \bar{Y}_{x}, \quad Y^{*}=Y_{* x} \oplus \bar{Y}_{* x}
$$

При этом $\bar{Y}_{* x}=\operatorname{im} \bar{P}_{x}^{*}=Y_{x}^{\perp}, Y_{x}=\operatorname{im} P_{x}$. Для $x$ из окрестности точки $x_{0}$, определяемой неравенством $\left\|P_{x}-P_{x_{0}}\right\|<1$, подпространства $Y_{x}, Y_{* x}$ изоморфины соответственно подпространствам $Y_{x_{0}}, Y_{* x_{0}}$. Это вытекает из следующей леммы (см. [3]).

Лемма. Пусть $P, Q: Z \rightarrow Z$ - проекторь банахова пространства $Z$ на его подпространства $X=\operatorname{im} P, Y=\operatorname{im} Q$. Ecли $\|P-Q\|<1$, mо подпространства $X$ и $Y$ изоморфнь, причем сужение $Q \mid X: X \rightarrow Y$ есть изоморфизм.

Заметим еще, что для реффлексивного пространства $Y$ достаточно требовать, чтобы существовало семейство проекторов $P_{* x}$ на $Y_{* x}$. В случае конечной мультикосимметрии $L=\left\{L_{1}, \ldots, L_{k}\right\}$ регулярность точки $x_{0} \in X$ означает попросту, что 1-формы $L_{1} x_{0}, \ldots, L_{k} x_{0}$ линейно независимы.

2. Локальные многообразия решений косимметрических уравнений. Будем говорить, что линейный оператор $T: X \rightarrow Y$, где $X, Y$ - банаховы пространства, регулярно разрешим, если он нормально разрешим по Хаусдорфу $\left(\operatorname{im} T=\left(\operatorname{ker} T^{*}\right)^{\perp}\right)$ и его ядро дополнимо в $X$. Тогда сужение оператора $T$ на любое дополнение его ядра есть изоморфизм этого дополнения и $\operatorname{im} T$. 
ТЕОРема 1. Пусть в окрестности решения $x_{0}$ уравнения (3) оператор $F$ непрерьвно дифференцируем. Предположим, что $F^{\prime}\left(x_{0}\right)$ - регулярно разрешимый оператор, $x_{0}$ - регулярнал точка мультикосимметрии (2) оператора $F$ и выполняется условие

$$
\operatorname{ker}{F^{\prime *}}^{*}\left(x_{0}\right)=Y_{* x_{0}}
$$

Тогда мнохсество решений уравнения (3) в некоторой окрестности точки $x_{0}$ представляет собой подмногообразие $E$ в $X$, касающееся в $x_{0}$ подпространства $X_{0}=\operatorname{ker} F^{\prime}\left(x_{0}\right)$. Точнее, на некоторой окрестности $\Sigma$ нуля в $X_{0}$ определено непрерывно дифференцируемое вложение $\varphi: \Sigma \rightarrow X$ $u E=\operatorname{im} \varphi$. Eсли $F \in C^{k}$ при некотором $k=1,2, \ldots, \infty, \omega$, то $и \varphi \in C^{k}$.

Заметим, что условие (6) означает отсутствие дополнительных вырождений, помимо тех, которые вызваны косимметриями.

ДоКАЗАТЕЛЬСТво получается сведением к классической теореме о неявной функции посредством двух редукций - левой и правой. Во-первых, заметим, что уравнение (3) эквивалентно уравнению (левая редукция)

$$
\bar{P}_{x_{0}} F x=0
$$

в окрестности точки $x_{0}$, определяемой неравенством $\left\|P_{x}-P_{x_{0}}\right\|<1$. Действительно, (2) очевидно влечет (7), а из уравнения (7), записанного в эквивалентной форме $F \boldsymbol{x}=\left(P_{x_{0}}-P_{x}\right) F x$, следует (2).

Далее полагаем (правая редукция)

$$
x=x_{0}+s+\bar{x}, \quad s=Q\left(x-x_{0}\right), \quad \bar{x}=\bar{Q}\left(x-x_{0}\right)
$$

где $Q: X \rightarrow X, \operatorname{im} Q=X_{0},-$ проектор, существуюший ввиду регулярной разрешимости оператора $F^{\prime}\left(x_{0}\right)$. Тогда уравнение (7) приводится к виду

$$
\Phi(\bar{x}, s)=0, \quad \Phi(\bar{x}, s) \stackrel{\text { def }}{=} \bar{P}_{x_{0}} F\left(x_{0}+s+\bar{x}\right) .
$$

Оператор $\Phi: \bar{X}_{0} \times X_{0} \rightarrow \bar{Y}_{x_{0}}, X_{0}=\operatorname{im} \bar{Q}$, определен в некоторой окрестности нуля пространства $\bar{X}_{0} \times X_{0}$. Доказательство теоремы 1 теперь получается применением классической теоремы о неявной функции к уравнению (3).

Естественно встает вопрос о продолжении локального подмногообразия решений уравнения (3). Содержащая его компонента связности $S$ множества решений вдали от точки $x_{0}$ может быть устроена по существу произвольно. Если же компонента $S$ регулярна (целиком состоит из регулярных, удовлетворяющих условиям теоремы 1 , точек) и компактна, то $S$ - замкнутое подмногообразие. Поскольку на $S$ определена нигде не исчезающая пфаффова форма (косимметрия), его эйлерова характеристика равна нулю. Отсюда следует, что регулярное и компактное подмногообразие решений в случае, когда оно двумерно, есть либо тор $T^{2}$, либо бутылка Клейна $K^{2}$, а среди сфер $S^{n}$ возможны лишш $S^{1}, S^{3}, S^{7}$ [4]. Сушествование многих косимметрий налагает на $S$ дальнейшие топологические ограничения. 
3. Косимметрические уравнения с параметром. Рассмотрим теперь уравнение с векторньм параметром $\lambda$ :

$$
F(x, \lambda)=0 .
$$

Здесь $F: X \times \Lambda \rightarrow Y$ - заданный оператор, $X, \Lambda, Y$ - вещественные банаховы пространства. Введем банаховы пространства $\widehat{X}=X \oplus \Lambda, \widehat{Y}=Y \oplus \Lambda$ и оператор $\widehat{F}: \widehat{X} \rightarrow \widehat{Y}$, полагая для любого $\widehat{x}=(x, \lambda) \in \widehat{X} \widehat{F} \widehat{x}=(F(x, \lambda), 0)$. Тогда уравнение (9) примет вид

$$
\widehat{F} \widehat{x}=0
$$

причем память о происхождении этого уравнения выражается в существовании стандартной мультикосимметрии $\Lambda_{\Lambda}=\left\{L_{\psi}\right\}_{\psi \in \Lambda^{*}}$ с множеством индексов $\Lambda^{*}$; по определению, $\Lambda_{\psi}(x, \psi)=(0, \psi)$.

Tеорема 2. Пусть $F\left(x_{0}, \lambda_{0}\right)=0$ для некоторых $x_{0} \in X, \lambda_{0} \in \Lambda, u$ для любого $\lambda$ из некоторой окрестности точки $\lambda_{0}$ отобрахение $F_{\lambda}: X \rightarrow Y$ : $x \mapsto F(x, \lambda)$ допускает мультикосимметрию (2), не зависящую от параметра. Предположим, что для уравнения $F\left(x, \lambda_{0}\right)=0$ выполнены предполохсения теоремы 1: частная производная $F_{x}{ }^{\prime}\left(x_{0}, \lambda_{0}\right): X \rightarrow Y-$ регулярно разрешимый оператор, $x_{0}$ - регулярная точка мультикосимметрии $L, u$ отсутствуют дополнительные вырохдения, так что

$$
\operatorname{ker} F_{x}{ }^{*}\left(x_{0}, \lambda_{0}\right)=Y_{* x_{0}}
$$

где $Y_{* x_{0}}-$ замкнутая линейная оболочка мнохсества функиионалов $L_{m} x_{0}$.

Тогда существуют окрестности $\Delta$ точки $\lambda_{0}$ в $\Lambda$ и $\Omega$ точки $x$ в $X$ такие, что мнохсество всевозмохных решений $\widehat{x}=(x, \lambda) \in \Omega \times \Delta$ уравнения (9) есть дифференцируемое подмногообразие в $X \times \Lambda$, касательное $\kappa$ подпространству $X_{0} \times \Lambda$ пространства $X \times \Lambda$ в точке $\left(x_{0}, \lambda_{0}\right) ;$ здесь $X_{0}=\operatorname{ker} F^{\prime}{ }_{x}\left(x_{0}, \lambda_{0}\right)$. Точнее, для всех $\lambda \in \Delta$ всякое решение уравнения (8) относительно $x$, располохенное в окрестности $x_{0}$, имеет вид $x=x_{0}+s+\theta(s, \lambda)$, где $s-$ произвольная точка $X_{0}$ с достаточно малой нормой; отобрахсение $\theta: \Omega \times \Delta \mapsto X C^{1}$-гладко $u \theta^{\prime}{ }_{s}\left(0, \lambda_{0}\right)=0$. Eсли $F \in C^{k}, k=1,2, \ldots, \infty, \omega$, mo $u \theta \in C^{k}$.

ДокАЗАТЕЛЬСтво получаем, переходя от уравнения (9) к уравнению (10) и применяя к последнему теорему 1. При этом уравнение (10) допускает косимметрию $\widehat{L}=L \cup L_{\Lambda}$, получаемую присоединением к заданной мультикосиметрии $L$ стандартной мультикосимметрии $L_{\Lambda}$.

Любопытно, что при таком подходе к лассическая теорема о неявной функции для уравнения (9) с параметром оказьвается частным случаем ее косимметрической версии для уравнения (11) без параметра; в принципе, можно построить доказательство так, чтобы избежать здесь порочного круга.

Скажем несколько слов о возможных обобщениях понятия косимметрии. Если $X$ и $Y$-многообразия и $F: X \rightarrow Y$-отображение, то назовем соотношением для $F$ отображение $\Phi: X \times Y \rightarrow \mathbb{R}$ такое, что $\Phi(x, F x)=0$, причем $\Phi(x, 0)=0$ для всех $x \in X$. Уравнение (1) в случае, когда $X$ и $Y$ - банаховы пространства, получаем, когда $\Phi(x, y)=(y, L x)$.

Далее, пусть $M_{X}$ - многообразие, моделируемое банаховым пространством $X$, и $V$ - векторное расслоение с базой $M_{X}$, проекцией $\pi: V \rightarrow M_{X}$ и слоем $Y[5]$. 
Если $F: M_{X} \rightarrow V$ - сечение расслоения $V$, то назовем его косимметрией сечение $L: M_{X} \rightarrow V^{*}$ сопряженного расслоения такое, что $(F m, L m)=0$ для всех $m \in M_{X}$. Можно рассматривать и расслоения $V$ с произвольной базой, не обязательно $M_{X}$.

4. Продолжение решений по параметру. Пусть при некотором $\lambda=\lambda_{0}$ уравнение (9) имеет замкнутое изолированное множество $E \subset X$ решений. Скажем, что $\lambda_{0}$ - глобально регулярное значение параметра $\lambda$ для множества $E$, если существуют окрестность $\Delta$ точки $\lambda_{0}$ в $\Lambda$ и окрестность $\Omega$ множества $E$ в $X$ такие, что множество решений $(x, \lambda) \in \Omega \times \Delta$ уравнения (9) диффеоморфно $E \times \Delta$. Нерегулярное значение будем также назьвать бифуркационным.

Если $F\left(x_{0}, \lambda_{0}\right)=0$, то назовем $\lambda_{0}$ локально резулярным в точке $x_{0}$ значением параметра, если существуют окрестности $\Omega_{0}$ точки $x_{0}$ в $X$ и $\Delta_{0}$ точки $\lambda_{0}$ в $\Lambda$ такие, что множество решений уравнения $F(x, \lambda)=0$ в $\Omega_{0} \times \Delta_{0}$ диффеоморфно $E_{0} \times \Delta_{0}$, где $E_{0}-$ множество решений уравнений $F\left(x, \lambda_{0}\right)=0$ в $\Omega_{0}$.

ПРЕДЛОЖЕНИЕ 2. Пусть уравнение (9) при $\lambda=\lambda_{0}$ имеет в $X$ компактное, замкнутое, связное и регулярное подмногообразие решений $E_{0}$, моделируемое подпространством $X_{0} \subset X$. Тогда $\lambda_{0}$ - глобально регулярное значение параметра $\lambda$ для подмногообразия $E_{0}$. Если же $\lambda_{0}-$ бифуркаиионное значение для подмногообразия $E_{0}$, то существует точка $x_{0} \in E_{0}, \boldsymbol{\theta}$ которой $\lambda_{0}$ не является локально регулярным.

E-mail: yudovich@ns.unird.ac.ru

Поступило 04.01 .96

\section{СПИСОК ЦИТИРОВАННОЙ ЛИТЕРАТУРЫ}

1. Юдович В. И. // Матем. заметки. 1991. Т. 49. № 5. С. 142-148. 2. Yudovich V. // Chaos. 1995. V. 5. № 2. Р. 402-411. 3. Глазман И. М., Любич Ю. И. Конечномерньй линейньй анализ в задачах. М.: Наука, 1969. 4. Шварц Дж. Дифференциальная геометрия и топология. М.: Мир, 1970. 5. Ленг С. Введение в теорию дифференцируемых многообразий. М.: Мир, 1967. 